\title{
INDUSTRIAL ROBOT AUTOMATION IN SOLVING NON-VACUUM ELECTRON-BEAM WELDING PROBLEMS
}

\author{
Vasiliy Borovik ${ }^{1,3}$, Vladislav Shatravin ${ }^{1,3}$, Il'jas Junusov ${ }^{2}$, Dmitri Shashev ${ }^{3}$, \\ Sergei Kornilov ${ }^{1}$, Nikolai Rempe ${ }^{1}$, Stanislav Shidlovskiy ${ }^{2,3, a}$ \\ ${ }^{1}$ Tomsk State University of Control Systems and Radioelectronics, 634050, Tomsk, Russia \\ ${ }^{2}$ National research Tomsk polytechnic university, 634050, Tomsk, Russia \\ ${ }^{3}$ National research Tomsk state university, 634050, Tomsk, Russia
}

\begin{abstract}
The paper describes one modeling automated hardware-software system to develop additive technologically - based products. Algorithm synthesis for industrial robot control was performed. In this case, the operating tool is an electron -beam projector with plasma emitter. The model testing results of thermal processes (in 3D-enqueuing) proceeding within additive substrate material system have been described. The modeling results revealed those required parameters of emission power which would provide the melting of additive material (TiC) excluding boiling.
\end{abstract}

\section{Introduction}

As great emphasis is placed on such factors as production rate and cost, the application of additive technologies in developing industrial products furthers the possible implementation of economically- effective methods in the production of items based on consumable patterns. This technology implements the basic principles of developing new-generation materials and embraces an innovative approach in designing and producing updated products comparable to the conventional methods, i.e. casting and mechanical metalcutting.

This paper describes one method in architecturing automated hardware-software system for designing additive technologically-based $[1,2]$ items. This system includes electronbeam projector with plasma emitter, industrial robot and hardware-software appliance ensuring mutually-agreed interaction of the whole system itself. Electron-beam projector with differential-pumping system [3], intended for non-vacuum material processing and operating as a tool for the industrial robot $[4,5]$. The latter also provides the positioning of the electron-beam projector relative to the workpiece.

\footnotetext{
${ }^{a}$ Corresponding author: shidlovskiysv@mail.ru
} 


\section{Electron-beam projector with plasma emitter}

Low-voltage gas discharge mode - hollow-cathode reflective discharge [6] is used in projectors with plasma emitter which are designed to generate focused high-brightness Ebeams. Electrons exposed to electric field are emitted from the gas-discharge plasma [6].

The basic projector structure unit is the metal-ceramic unit - discharge chamber (Figure1, $a$, marked region).

Discharge generates within the discharge cell, which, in its turn, includes internal hollow cathode surfaces (3), cylindrical anode (4) and emitting cathode (1) (Figure1, a). Axially-symmetric field including the axial induction of about $0.1 \mathrm{~T}$ is formed between the hollow and emitting cathodes of the permanent magnet.

Plasma-supporting gas pressure in the discharge chamber is approximately $5 \cdot 10^{-2}$ Torr. The same pressure of about $10 \div 20 \mathrm{~cm}^{3} \mathrm{~atm} / \mathrm{h}$ is created by dosed gas puffing supplied through the channel into the hollow cathode and pumped into the axial channel of emitting cathode. The glowing discharge is continuous under the voltage of $350 \div 450 \mathrm{~V}$ and current from 0.1 to $0.5 \mathrm{~A}$. This discharge current range renders electron beam of one to several hundred milliamphere.

Electron emission from the plasma occurs through the orifice in the emitting cathode of discharge chamber to accelerating field zone between this cathode and grounded highvoltage anode (extractor).

Produced electron beam in the accelerating gap is focused by the magnetc field and transported to the vacuum chamber [7] and / or atmosphere [3].
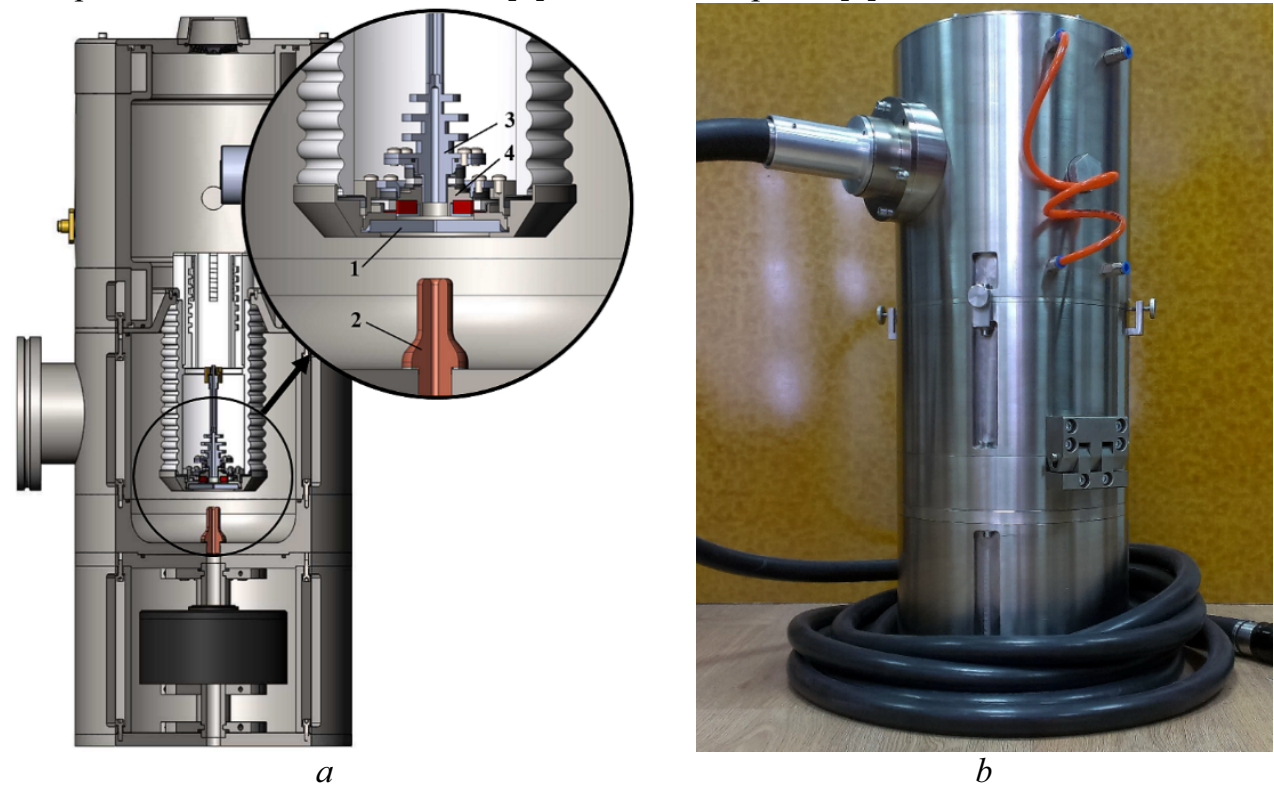

Figure 1. Electrode diagram (a) and external view (b) of the projector with plasma emitter [8]: 1 - emitting cathode; 2 - extractor; 3 - hollow cathode; 4 - anode.

External projector view, application design designed with beam extractor in atmosphere (Figure $1, b$ ). This projector produces focused electron beams of $120 \mathrm{keV}$ and current up to $200 \mathrm{~mA}$. 


\section{Automated industrial robot}

3D printing involves designing the actual (physical) product based on its digital model. Such a model could be a standard component designed within the framework of solid modeling and printed to STL. STL format is a 3D model as a list representation of triangular faces describing its surface, as well as its standard specifications. Further applying the programming support "slicer" the model is transformed into G-code, representing the vocabulary for $3 \mathrm{D}$ printer and relevant Cartesian coordinates. Therefore, manipulator movement algorithm involves Cartesian coordinates and G-code commands as initial data, i.e. to solve specific instance of inverse kinematic problems.

Let's consider $3 \mathrm{DOF}$ robotic manipulator. The simpliest calculation is the geometrical method in solving inverse kinematic problems [9].

The first problem solution step is selecting initial manipulator position, including first manipulator link (segment) perpendicular to the earth; second link (segment) - straight along the axis $O X$. Thus, initial-values of related manipulator angles are:

$$
\alpha_{0}=0^{\circ}, \beta_{0}=90^{\circ}, \gamma_{0}=0^{\circ} \text {, }
$$

where, $\alpha_{\mathrm{i}}-$ second link angle of rotation to axis $O X$ in the plane parallel to $O X Y ; \beta_{\mathrm{i}}-$ angle between horizontal plane surface of installed manipulator and first link angle after movement in $i$ - point; $\gamma_{i}$ - angle between first link angle and second link angle after movement in $i$-point.

Lateral view of the horizontal plane movement axis is denoted $O U$, where the points lie in the Pythagorean theorem:

$$
u=\sqrt{x^{2}+y^{2}} ; \forall x \in X ; \forall y \in Y
$$

Vertical axis - axis $O Z$ of initial data.

Initial point- starting first manipulator link. The fact that the manipulator is installed on a mobile base will be considered in the addition of correction coefficients to obtained result.

Consequently, the point of manipulator gripper is described by the top-view coordinates $\left(x_{i}, y_{i}\right)$, of which the side-view angles $\alpha_{\mathrm{i}}$, and $\left(u_{i}, z_{i}\right)$ are determined, and, whereof angles $\beta_{\mathrm{i}}$ and $\gamma_{i}$ are calculated. It is obvious that the initial point of the gripper could be determined in terms of the manipulator link length. So, the first link length $l_{1}=1350 \mathrm{~mm}$, second link $l_{2}$ $=1500 \mathrm{~mm}$. In this case, the initial point has the following top-view coordinates $(1500 ; 0)$ and side-view coordinates $(1500 ; 1350)$.

Let's consider the top-view (Figure 2).

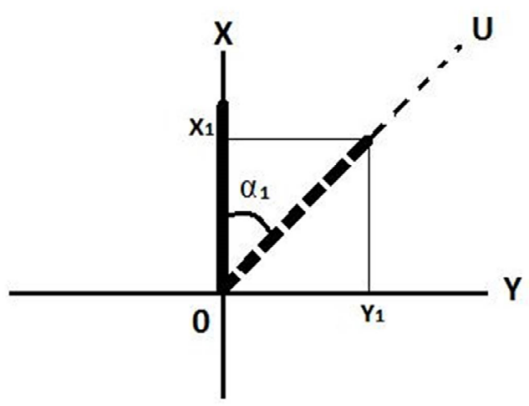

Figure 2. Coordinate system $X Y$.

It is evident that according to the Pythagorean theorem moving from the initial point $\left(x_{0}, y_{0}\right)$ to point $\left(x_{1}, y_{1}\right)$, the manipulator rotates through an angle $\alpha_{1}$ of system defined: 
or, transforms into:

$$
\left\{\begin{array}{l}
\sin \left(\alpha_{i}\right)=x_{i} / \sqrt{a^{2}+y^{2}}, \\
\cos \left(\alpha_{i}\right)=y_{i} / \sqrt{a^{2}+y^{2}},
\end{array}\right.
$$

$$
\sin \left(\alpha_{i}\right)-\cos \left(\alpha_{i}\right)=\left(x_{i}-y_{i}\right) / \sqrt{x^{2}+y^{2}} .
$$

The problem-solving result of this system is the sought-for angle $\alpha_{1}$.

Let's consider the side-view movement in plane OUZ (Figure 3).

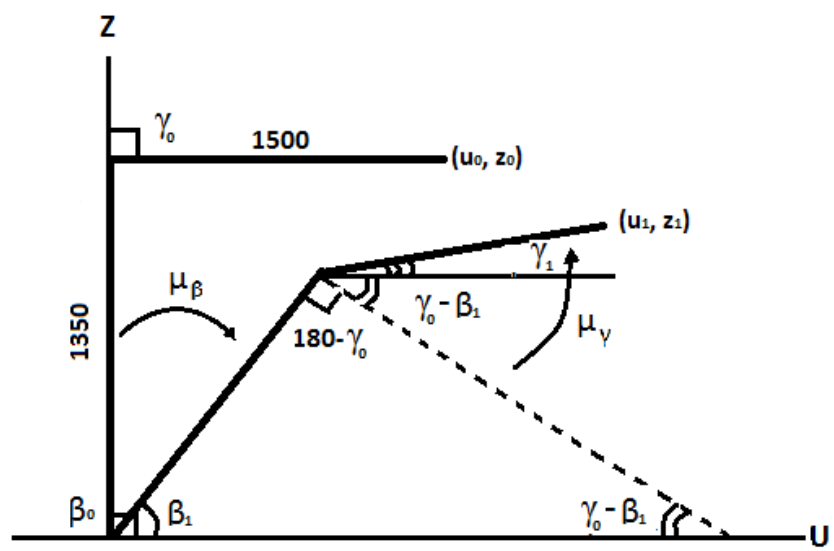

Figure 3. Coordinate system $Z U$.

Let us assume that the manipulator is moved to a point $\left(u_{1}, z_{1}\right)$. As is seen from the above diagram to simplify the calculations the movement links are divided into two steps:

1) rotation of first link to an angle $\mu_{\beta}$ relative to the origin of the coordinates;

2) rotation of second link to an angle $\mu_{\gamma}$ relative to the conjugation point of the first link.

To determine these angles the following algorithm is used: the dependence of each coordinate to the finite point of each link is described through the length of these links $l_{1}$ and $l_{2}$ and angles $\beta_{i}$ and $\gamma_{i}$. The following system of nonlinear equations was calculated:

$$
\left\{\begin{array}{l}
l_{1} \cos \left(\beta_{i}\right)+l_{2} \cos \left(\gamma_{i}\right)=u_{i}, \\
l_{1} \sin \left(\beta_{i}\right)+l_{2} \sin \left(\gamma_{i}\right)=z_{i} .
\end{array}\right.
$$

The solution of one system involves the angles $\beta_{i}$ and $\gamma_{i}$. However, according to selected algorithm, $\beta_{i}$ and $\gamma_{i}$ are not angles to $\mu_{\beta}$ and $\mu_{\gamma}$; but, based on this fact and using the initial values of adjoint angles $\beta_{0}$ and $\gamma_{0}$ it can be expressed by:

$$
\begin{aligned}
& \mu_{\beta}=\beta_{0}-\beta_{1}, \\
& \mu_{\gamma}=\gamma_{0}-\beta_{1}+\gamma_{1} .
\end{aligned}
$$

The links, moving to the calculated angles $\mu_{\beta}$ and $\mu_{\gamma}$, center the gripper in the specified point. It is obvious that this algorithm could be iterative and one could calculate as many points as possible. However, it is necessary to recalculate the angle $\gamma_{i}$ after each iteration, accordingly to the following relation:

$$
\gamma_{i}=\gamma_{i-1}-\mu_{\gamma}
$$

This is due to the fact that the system solution is the internal narrow angles between the links, and, according to specified definition, this angle is counted clockwise from the axis of the previous link. 
The main disadvantage of described algorithm is the strong dependency of system solution equation results to the initial approximation task. To obtain correct results it is necessary to make an appropriate assumption of the angle dimension at the initial stage.

Obtained control algorithm is implemented in Matlab and applying extension packs of Simscape Multibody. Accuracy testing of examined algorithm results was conducted with consideration of original effector position gravity center. The research results showed that the industrial robot ensures the positioning of the operating tool pertaining to specified trajectory and required accuracy.

\section{Modeling temperature setting}

To produce highly-qualified "grown" items it is necessary not only to provide the required penetrating beam trajectory, but also to consider the electron beam energy intensity and velocity, which, in its turn, depends on the thermal-physical properties of used sources.

To produce an industrial item non-vacuum printers should have such electron beam parameters that would optimally further the melting of additive metal powder during the production process itself. In this case, the behaviour of additive material in melting, temperature range of penetrating electron beam and crystallization velocity of used material should be examined.

The mathematical heat transfer model of porous medium and its phase transitions could be written as $[10,11]$ :

$$
\Psi(T) \frac{\partial T}{\partial t}=k\left(\varepsilon_{V}, \varepsilon_{\alpha}\right) \nabla^{2} T+U(q)
$$

where $\Psi$ - dimensionless effective heat capacity, including latent heat release of phase transition; $T$ - absolute temperature; $t$ - time; $k$ - temperature conductivity; $\varepsilon_{V}$ and $\varepsilon_{\alpha}$ - porosity characteristics of powder-coated layer; $U$ - thermal intensity of volume source, related to beam power .

Geometric computational region is depicted in Figure 4.

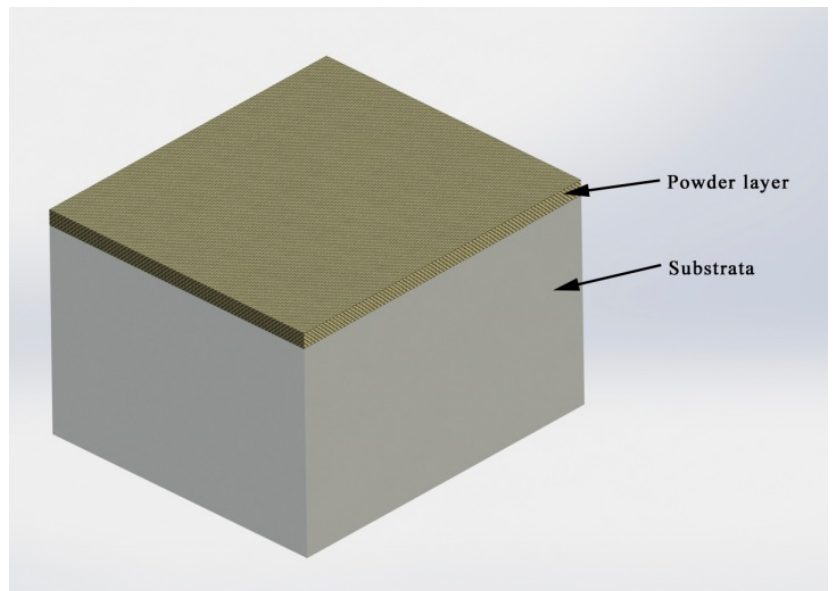

Figure 4. Computational region.

Described fuse layer is titanium carbide powder (TiC) including such properties as [12]: Thermal capacity in solid phase, $J /(g K)$ :

$C_{p}(T)=\left(-1.608 \mathrm{e}-18 T^{6}+1.923 \mathrm{e}-14 T^{5}-9.185 \mathrm{e}-11 T^{4}+2.234 \mathrm{e}-07 T^{3}-0.0002895 T^{2}+\right.$ $0.1953 T-3.611) / 59.911$.

Thermal capacity in liquid phase, $J /(g K): C_{p_{-} l i q}=1.0476$. 
Melting temperature, $K: T_{\text {liq }}=3290$.

Latent heat of melting $\mathrm{J} / \mathrm{kg}: L_{1-2}=1.1873$.

Density, $\mathrm{g} / \mathrm{cm}^{3}: \rho=4.93$.

Powder-coated layer thickness is $0.2 \mathrm{~mm}$. Substrata is of stainless steel, thickness ranging from 1 to $7 \mathrm{~mm}$.

Control sintering by electron beam exposure includes the following parameters: 1) actual emission power; 2) beam velocity.

Emission rate depends on the following factors: melt temperature of material powder, thermal conductivity, size and shape of powder particles so as to prevent overheating and further melt boiling. Generally, distribution of beam power is:

$$
\begin{gathered}
J(x, y)=F(x) F(y) W N, \\
F(r)=\frac{1}{\sqrt{2 \pi} \sigma} \exp \left(\frac{-r^{2}}{2 \sigma^{2}}\right) \\
N=(\sqrt{2 \pi} \sigma)^{2} \\
W=J_{0} V \\
J_{0}=\frac{I}{2 \sigma^{2} \pi}
\end{gathered}
$$

where, $\sigma$ - effective beam range, $\mathrm{m} ; I$ - electron beam current ; $V$ - voltage, $110 \mathrm{kV} ; x, y-$ Cartesian coordinates (cross-shaped $\mathrm{X} / \mathrm{Y}$ axis), the normal of which corresponds to electron beam symmetry axis.

Figure 5 presents the distribution of beam power in the described projector.

Figure 5. Beam power distribution.

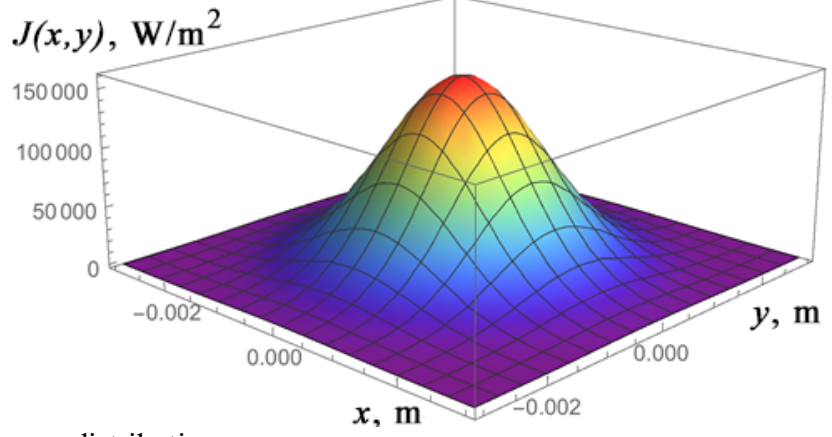

Beam penetration coefficient is characterized by the depth penetration into the powdercoated layer and depends on the powder porosity (shape and size of particles) and radiation surface absorption of powder particles.

Beam velocity is based on the melt temperature: beam velocity decreases for refractory materials and, reversely, increases for low-melting materials.

Modeling electron beam velocity trajectory (based on the results stated in section 3 ) it is necessary to apply correction for (7):

$$
J(x, y, t)=F\left[x+f_{x}(t)\right] F\left[y+f_{y}(t)\right] W N,
$$

where, $f_{x}(t)$ and $f_{y}(t)$ - dynamic functions, defining the changing position of electron beam symmetry axis relative to the origin of coordinates along $x$ and $y$ axes, respectively.

Defined equation including initial-boundary conditions is solved numerically, i.e. computer-calculated. In calculating the partial differential equation finite element method is used [8, 13-15]. Applying FDM (finite difference method) in problem-solving of solid 
bodies is represented as a sum of nodes. Substituting partial differential coefficients by corresponding finite differences the system of linear algebraic equations (SLAE) is derived. Visualized distribution is obtained on the basis of $I=15 \mathrm{~mA}$ and time $t=0.5 \mathrm{~s}$.

Figures 6 and 7 show that provided parameters and time of electron beam exposure ensure the melting of the powder-coated layer and upper region of substrata (backing).

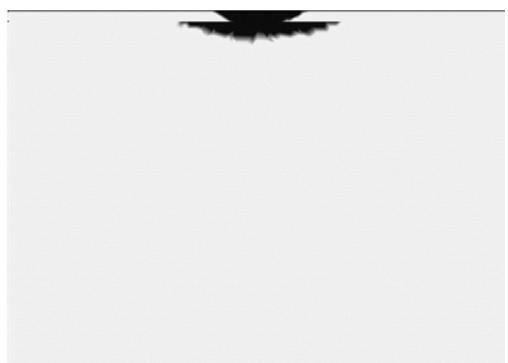

Figure 6. Fields of phase state: black «liquid», gray- «solid».

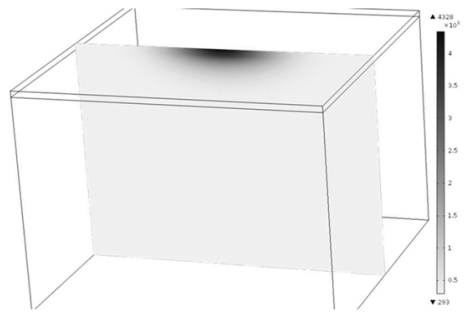

Figure 7. Temperature pattern.

Providing current range of beam within 7.5 -9.5 $\mathrm{mA}$ the functional dependency is obtained, reflecting the required electron beam exposure time for melting the powdercoated layer thickness of $0.2 \mathrm{~mm}$ and excluding boiling (Figure 8 ).

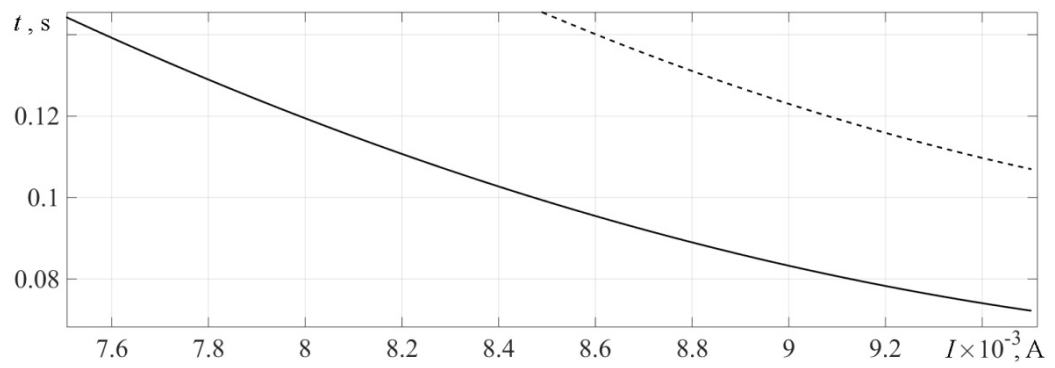

Figure 8. Electron beam current- beam exposure time dependency, providing the melting of the material: continuous line

$t_{m}^{\prime}(I)$ - for points on the powder-coated layer surface; dotted line $t_{m}^{\prime \prime}(I)$ - for points on the separation interface of powder-coated layer / substrata

Based on the modeling results the analytical expression for functional dependency is written (Figure 8), at $I \in[0,0075 ; 0,0095] \mathrm{A}$ :

$$
t_{m}^{\prime}(I)=9429 I^{2}-196,5 I+1,088, t_{m}^{\prime \prime}(I)=1,2 \cdot 10^{4} I^{2}-254 I+1,437 .
$$

\section{Conclusion}

The research revealed the thermal processes within the item during non-vacuum electron beam welding and automated robot control positioning the electron beam in the process itself. The most important control parameters, i.e. beam velocity (scanning) and beam intensity were determined. It was identified that the specific welding regimes depend on the beam penetration coefficient, which, in its turn, depends on the preparation method of powder-coated layer. The modeling results revealed those required parameters of emission power which would provide the melting of additive material excluding boiling. 


\section{Acknowledgment}

The following research has been financially supported by Ministry of Education and Science of the Russian Federation, Agreement № 14.577.21.0018 (RFMEFI57714X0018).

\section{References}

[1] A. Townsenda , N. Seninb, L. Blunta , R.K. Leachb, J.S. Taylord, Precision Engineering 46, 34 (2016) doi: 10.1016/j.precisioneng.2016.06.001

[2] I. Gibson, D.W. Rosen, B. Stucker, Additive Manufacturing Technologies: 3D Printing, Rapid Prototyping, and Direct Digital Manufacturing ( Springer, New York, 2014)

[3] A. Aksenov, S. Kornilov, M. Motorin, N. Rempe, D. Shashev, S. Shidlovskiy, E+E 5-6, $129(2016)$

[4] Y. Zeng, W. Tian, W. Liao, Robotics and Computer-Integrated Manufacturing 42, 113 (2016) doi: 10.1016/j.rcim.2016.05.011

[5] R. Lia, Y. Zhao, Measurement 88, $113 \quad$ (2016) doi: 10.1016/j.measurement.2016.02.038

[6] V.I. Gushenets, E.M. Oks, G.Yu. Yushkov, N.G. Rempe, Laser and Particle Beams 21, 123 (2003) doi: 10.1017/S0263034603212027

[7] T. Hassel, N. Rempe, S. Kornilov, A. Beniyash, Welding and Cutting 11, 122 (2012)

[8] S.Yu. Kornilov, N.G. Rempe, S.V. Shidlovskiy, Technical Physics 61, 841 (2016) doi: $10.1134 / \mathrm{S} 1063784216060116$

[9] T. Messaya, R. Ordóñeza, E. Marcilb, Robotics and Computer-Integrated Manufacturing 37, 33 (2016) doi: 10.1016/j.rcim.2015.06.003

[10] M. Krivilev, E. Kharanzhevski, G. Gordeev, V. Ankudinov, Large-scale Systems Control 31, 299 (2010)

[11] M.St. Węglowskia, S. Błachaa, A. Phillipsb, Vacuum 130, 72 (2016) doi: 10.1016/j.vacuum.2016.05.004

[12] M.W. Jr. Chase, NIST-JANAF Thermochemical Tables (American Inst. of Physics, New York, 1998)

[13] Y. Liu, Ch. Tian, T. Liu, F. Kong, MATEC Web of Conferences 34, 02014 (2015) doi: $10.1051 /$ matecconf $/ 20153402014$

[14] F. Klocke, D. Lung, H. Puls, Procedia CIRP 8, 240 (2013) doi: 10.1016/j.procir.2013.06.096

[15] S.V. Panin, V.I. Syryamkin, A.I. Glukhikh, Avtometriya (4), 79 (2003) 